\title{
Disparate Species-Dependent Leukocyte Behavior before and after Adding an Elastase Inhibitor
}

\author{
Nicholas J Cheronis $^{1 *}$, Nathan A Groathouse ${ }^{2 *}$, Richard A Bowen ${ }^{2}$, Sanford R Simon ${ }^{3}$, John C Cheronis ${ }^{1}$ and John E Repine ${ }^{*}$ \\ ${ }^{1}$ Paradocs Biomedical, Ltd., Breckenridge, CO, USA \\ ${ }^{2}$ Department of Biomedical Sciences, Colorado State University, Fort Collins, CO, USA \\ ${ }^{3}$ Department of Pathology and Biochemistry, State University of New York, Stony Brook, NY, USA \\ ${ }^{4}$ Webb-Waring Center and University of Colorado Denver Health Sciences Center, Aurora, CO, USA \\ *These authors contributed equally to this work
}

\begin{abstract}
Animal models are often used to identify aspects of the pathophysiology and to develop new therapeutic interventions for human disorders. Leukocyte serine elastases are key mediators of inflammation and meaningful therapeutic targets because of their potential contributions to the Acute Respiratory Distress Syndrome (ARDS) and other inflammatory disorders. We compared the extracellular matrix degrading and bactericidal activities against both Escherichia coli and Staphylococcus aureus, of leukocytes from humans, dogs, hamsters, rats, pigs and rabbits before and after adding highly selective serine elastase inhibitors. Not surprisingly, we found significant species-dependent differences in both activities and the responses to adding serine elastase inhibitors. Our results reinforce the need for care in selecting the optimal animal species for evaluating both the underlying pathophysiology of inflammatory diseases and potential new interventions being developed to treat these conditions.
\end{abstract}

Keywords: Extracellular matrix; Neutrophil elastase; Bactericidal activity; Proteinase-3

\section{Introduction}

The Acute Respiratory Distress Syndrome (ARDS) causes significant morbidity and mortality worldwide. Approximately 200,000 individuals develop ARDS per year in the US alone and these patients experience a mortality rate approaching 40\% [1-4] Along with trauma, among the most common predisposing causes for ARDS are severe bacterial pneumonias, systemic infections, and sepsis [5-8]. Furthermore, ARDS is the most common proximal cause of death accompanying pneumonias, sepsis and multiple organ failure [5-8] unfortunately, despite concerted efforts over the last 30 years to treat not only ARDS but also sepsis; no specific therapeutic intervention has been approved for treating either condition [9].

Paramount to the objective of developing interventions that can ameliorate ARDS is an appreciation that peripheral blood leukocytes, especially neutrophils, are critical to the development and progression of the alveolar-endothelial barrier degradation, interstitial edema, impaired gas exchange, and lung injury seen in patients with ARDS [5-8]. Evidence supporting this concept includes the observations that ARDS patients consistently have many neutrophils in their lungs and lung lavages, that are in fused radio-labeled autologous neutrophils rapidly accumulate in the lungs of ARDS patients, and that ARDS rarely occurs in the absence of neutrophils [6]. Numerous studies in animal models also support the role of neutrophils as contributors to the pathophysiology causing ARDS [10].

As essential mediators of an acute inflammatory response, leukocytes also function to contain and eradicate both gram negative and gram positive bacteria. Leukocyte bactericidal activity is mediated by a complex interplay of naturally occurring bactericidal proteins, defensins [11], reactive oxidant species, such as Hypochlorous Acid $(\mathrm{HOCl})$ and hydrogen peroxide $\left(\mathrm{H}_{2} \mathrm{O}_{2}\right)$ [12] and a variety of other enzymes [13]. Among the proteolytic enzymes implicated in phagocytic bactericidal activity are the serine elastases, neutrophil elastase and proteinase 3, that are primarily expressed in neutrophils, monocytes and macrophages [14-17]. Not surprisingly, these enzymes are also purported to play a critical role in the underlying pathophysiology of ARDS [5-7,18-23]. Despite underlying concerns that inhibiting these enzymes will impair pathogen clearance - a concern that has contributed to the reticence of some biopharmaceutical companies to pursue interventions targeting these enzymes -leukocytes and serine elastases remain prime targets for investigating and developing therapeutic interventions for ARDS.

Clearly, animal models of ARDS are needed to evaluate both the injurious as well as beneficial (antimicrobial and other) activities of leukocytes and the response to serine elastase inhibitors are needed to advance the development of much needed interventions for ARDS and sepsis. In this regard, a recent, provocative report suggests that at least with respect to acute inflammatory processes, the genomic responses of mice poorly mimic the genomic responses of human subjects [24]. Their observation begins to illustrate the potential difficulties of extrapolating the findings of animal models of inflammatory diseases from other species to humans. However, their study only predicted that the inflammatory actions of human and mice might be different and respond differently to pharmaceutical interventions without actually demonstrating this possibility. Regardless, their findings underscore emerging concerns about how potential therapeutics are evaluated in non-clinical settings and emphasize the diligence needed to more rationally select and justify the appropriateness of animal models that are used to direct the development of interventions intended for combating human diseases.

In the present investigation, we compared the activities of leukocytes derived from human subjects as well as five other mammalian species. Our goal was to begin to assess species-dependent differences in leukocyte function and to identify optimal animal

*Corresponding author: John E Repine, M.D., Webb-Waring Center. 12850 East Montview Blvd., Aurora, CO 80045, USA, Tel: 303-724-4788; E-mail: John.Repine@ucdenver.edu

Received July 29, 2013; Accepted August 28, 2013; Published August 30, 2013

Citation: Cheronis NJ, Groathouse NA, Bowen RA, Simon SR, Cheronis JC, et al. (2013) Disparate Species-Dependent Leukocyte Behavior before and after Adding an Elastase Inhibitor. J Pulm Respir Med 3: 155. doi:10.4172/2161-105X. 1000155

Copyright: (c) 2013 Cheronis NJ, et al. This is an open-access article distributed under the terms of the Creative Commons Attribution License, which permits unrestricted use, distribution, and reproduction in any medium, provided the original author and source are credited. 
species for the development and testing of serine elastase inhibitors as human therapeutics. This objective reflects the continuing need for being able to conduct enabling experiments in animals that cannot be done initially in humans and/or in expensive, complex human clinical trials. Our results indicate that there are appreciable species differences in leukocyte function before and following addition of serine elastase inhibitors that need to be considered when looking for the optimal animal species with which to evaluate potential novel therapeutic interventions and when the results from pre-clinical studies involving these species are used to predict potential outcomes in human subjects.

\section{Materials and Methods}

\section{Source of reagents}

Most of the reagents used were obtained from Sigma Chemical Company (St. Louis, Mo). However, we also synthesized two highly specific serine elastase inhibitors: CE-2072 [25] and AI-158 (collectively, SEI) for use in our studies (Figure 1 and Table 1). Both inhibitors penetrate intracellularly into human leukocytes and inhibit neutrophil elastase and proteinase-3at concentrations greater than or equal to 0.3 $\mu \mathrm{m}$ both in vitro and in vivo (data not shown).

\section{Leukocyte isolation}

Approval for these investigations was obtained from the animal and human subjects research committees of Colorado State University.

Fresh human whole blood was obtained from human volunteers and anti coagulated with EDTA. EDTA anticoagulated blood obtained from healthy individuals of the different species was obtained by venipuncture (dogs, pigs, rabbit) or cardiac puncture under ketaminexylazine anesthesia (rats and hamsters). The specific species tested were dogs (Canis lupus familiaris), pigs (Susscrofa domesticus), rabbits (Oryctolagus cuniculus), rats (Rattusnorvegicus, Sprague Dawley strain) and Syrian hamsters (Mesocricetus auratus).

For both Extracellular Matrix (ECM) degradation and bactericidal studies, leukocytes obtained from the peripheral blood of each species were assayed in parallel with and compared to peripheral blood leukocytes from normal human volunteers. Each comparison was
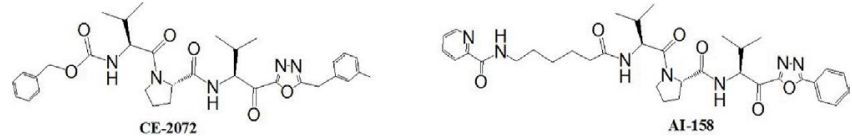

Figure 1: Chemical structures of serine elastase inhibitors (SEI), CE-2072 and Al-158.Both CE-2072 and Al-158 are potent inhibitors of neutrophil elastase (NE) and proteinase-3 (PR3) (Table 1). Importantly, for our studies, since both enzymes are stored in an active state and bound to proteoglycans in the azurophilic granules of neutrophils and monocytes/macrophages, both drugs have been demonstrated to penetrate intracellularly and inhibit their target enzymes [ $>90 \%$ inhibition] at concentrations exceeding $0.3 \mu \mathrm{M}$ (data not shown). Neither compound has any inhibitory activity against other serine proteinases, such as cathepsin G, trypsin, chymotrypsin, thrombin plasmin, human tissue plasminogen activator, nor any of the clotting factors at concentrations over 100 -fold higher than those used in our studies. Similarly, these compounds had no inhibitory activity, at any concentration, agains representative aspartyl-, cysteine- or metallo-proteinases

\begin{tabular}{|c|c|c|c|c|c|}
\hline Compound & $\begin{array}{c}\mathrm{K}_{\mathrm{i}}(\mathrm{NE}) \\
\mathrm{nM}\end{array}$ & $\begin{array}{c}\mathrm{K}_{\mathrm{i}}(\mathrm{PR} 3) \\
\mathrm{nM}\end{array}$ & $\begin{array}{c}\text { Solubility } \\
(\mathrm{PBS}, \mu \mathrm{M})\end{array}$ & $\begin{array}{c}\text { Plasma Protein } \\
\text { Binding }(\% \text { free })\end{array}$ & $\begin{array}{c}\text { Plasma Stability } \\
(\mathrm{t} 1 / 2 \text { minutes })\end{array}$ \\
\hline CE-2072 & 0.24 & 24 & $<10$ & 1.0 & $>100$ \\
\hline Al-158 & 0.29 & 6.2 & 69.0 & 18.0 & $>100$ \\
\hline
\end{tabular}

Table 1: Selected Biochemical and Physical Properties of the Serine Elastase Inhibitor (SEIs), CE-2017 and Al-158. conducted using two independent preparations of leukocytes per each species.

Leukocytes were prepared by initially mixing $4.5 \mathrm{ml}$ Polymorph prep (Axis-Shield PoC AS, Oslo, Norway) and $0.5 \mathrm{ml} \mathrm{HBSS}$ in a $15 \mathrm{ml}$ conical tube. Using a serological pipet, $5.0 \mathrm{ml}$ of EDTA anti coagulated whole blood was then carefully layered on top of the Polymorph prep mixture so as not to break the interphase barrier. Samples were next centrifuged at $500 \mathrm{x} \mathrm{g}$ for 30 minutes at $4^{\circ} \mathrm{C}$. Using a sterile suction pipette, the leukocyte fractions were carefully removed and combined to form the leukocyte mixtures used in each experiment.

Leukocytes were subsequently transferred into a clean $50 \mathrm{ml}$ conical tube, washed with $10 \mathrm{ml}$ cold $0.2 \% \mathrm{NaCl}$ for 45 seconds and then re suspended in $10 \mathrm{ml}$ of cold $1.6 \% \mathrm{NaCl}$. Samples were subsequently re centrifuged at $250 \mathrm{x}$ g for 5 minutes at $4^{\circ} \mathrm{C}$. The supernatants were then removed and discarded without disturbing the pellet. The wash steps were repeated and the leukocytes were again re centrifuged at $250 \mathrm{xg}$ for 5 minutes and the supernatants were discarded. The pellets were re suspended in $5 \mathrm{ml}$ cold HBSS (a final volume that was equivalent to the original whole blood starting volume). The re suspended pellet was placed on ice until used.

\section{Extracellular matrix (ECM) degradation}

We have selected for our work a complete native interstitial ECM synthesized by rat heart smooth muscle (R22) cells [26] in culture. R22 ECM, when cultured in the presence of ascorbic acid [27] consists of characteristic stromal or interstitial components (collagen I and III, elastin, proteoglycans, and fibronectin) and is virtually insoluble unless exposed to inflammatory cell proteases or $2 \mathrm{~N} \mathrm{NaOH}$. R22 ECM differs fundamentally from basement membranes, which are rich in type IV collagen and laminin, both absent in the interstitium. Addition of radio labeled precursors, such as proline, methionine-cystine, fucose, or sulfate, to the R22 growth medium induces selective labeling of specific ECM components, allowing assay of inflammatory cell degradative activity by release of soluble counts, an option not generally found in commercially available ECM preparations. By sequential enzyme digestion Rinehart et al. estimated that the R22 ECM composition to be $51 \%$ trypsin-sensitive (glycoproteins and proteins), 37\% collagenase sensitive (types I and III collagen), and 12\% human neutrophil elastase (HNE)-sensitive (elastin) [28].These results agree closely with those of Werb et al. [29] and Jones et al. [26].

The ECM was metabolically labeled with ${ }^{3} \mathrm{H}$-proline and ${ }^{35} \mathrm{~S}$-sulfate as previously described $[30,31]$ and the experiments were conducted in 48 well plates. First, $430 \mu \mathrm{l}$ of Hanks Balanced Salt Solution (HBSS) was added to each well followed by $20 \mu \mathrm{l}$ of a $500 \mu \mathrm{m}$ solution of CE2072 in HBSS/10\% DMSO or HBSS/10\% DMSO alone 30 minutes before adding a $50 \mu \mathrm{l}$ aliquot of the leukocyte suspension. Leukocytes were suspended in HBSS to provide a final concentration equivalent to $5 \times 10^{5}$ leukocytes/well. Subsequently, leukocytes were stimulated by adding either LPS+FMLP or heat-killed Staphylococcus aureus (HKS $100 \mu \mathrm{l}$ of a $1 \times 10^{8}$ organisms / $\mathrm{ml}$ in HBSS suspension) to represent leukocyte activation by either gram negative or gram positive bacteria, respectively. Incubation volumes were then adjusted by adding HBSS to achieve a final volume of $1 \mathrm{ml}$. LPS (serotype O127.88; Sigma) was pre incubated in human (or the appropriate animal) serum at $1 \mathrm{mg} / \mathrm{ml}$ for 30 minutes at $37^{\circ} \mathrm{C}$ and then serially diluted in HBSS before being added to wells at a final concentration of $1 \mu \mathrm{g} / \mathrm{ml}$. FMLP ( $\mathrm{N}$-formylMet-Leu-Phe; Sigma) was diluted from a stock concentration of $10 \mathrm{~mm}$ in DMSO and then added to achieve a final concentration in the wells of $10 \mu \mathrm{m}$. The final concentration of CE-2072, if present, was $10 \mu \mathrm{m}$. The HKS preparation used in our studies was a generous gift of Professor Charles Dinarello, MD of the University of Colorado. 
Leukocyte-ECM preparations were incubated for 6 hours at $37^{\circ} \mathrm{C}$ before careful removal of the supernatant medium. After removal of the supernatant, the remaining ECM was solubilized with $2.0 \mathrm{ml}$ of $2 \mathrm{M} \mathrm{NaOH}$. Supernatant and residual matrix radio activities were then measured using standard scintillation counting methods. Matrix degradation is expressed as the number of counts measured in the supernatant divided by the number of counts measured in the $\mathrm{NaOH}$ solubilized matrix x 100. All preparations were measured in triplicate resulting in 6 independent measurements for each condition and for each animal species. Since human leukocytes were used as controls for each of the species, data from a total of 30 measurements are reported for humans.

In preliminary studies, stimulus titration curves were conducted in order to identify the optimal concentration of each stimulus needed to produce a maximal effect for each species (data not shown). The concentrations used in our comparison studies were at least 3 fold (one half logarithms) higher than the plateau concentration that was observed to produce a maximal matrix degrading effect. For LPS + FMLP, the concentration response curves for human, dog, pig and rabbit leukocytes were comparable. However, a 10-fold increase over the concentrations of LPS +FMLP used for these species was needed to optimally activate the leukocytes of rats and hamsters. For HKS, the concentration response curves for human, dog and hamster leukocytes were comparable. However, no amount of added HKS would stimulate the leukocytes obtained from rat, pig and rabbit. Accordingly, for subsequent studies, the same amount of HKS was used for each of the six species.

\section{Bactericidal activity}

Escherichia coli and Staphylococcus aureus were obtained from frozen samples purchased from the ATCC and subsequently transferred into sterile $250 \mathrm{ml}$ conical culture tubes to which nutrient broth was then added to produce a final volume of $200 \mathrm{ml}$. Conical tubes containing the bacterial cultures were next placed into a shaking incubator at $37^{\circ} \mathrm{C}$ for 18 hours. Afterwards, bacterial cultures were transferred into $2 \times 100 \mathrm{ml}$ conical centrifuge tubes and centrifuged at $2000 \mathrm{rpm}$ for 15 minutes. The broth was then decanted and the tubes were filled with cold HBSS, vortexed and again centrifuged. After 2 centrifugations, the bacterial pellet was re suspended in HBSS and placed on ice until use.

Before use, bacterial suspensions were sonicated in a bath for 1015 seconds to disperse any clumped bacteria. To obtain the correct concentration of bacteria, $1.0 \mathrm{ml}$ of HBSS was placed into a cuvette to zero the spectrophotometer at $250 \mathrm{~nm}$. The bacterial suspension was subsequently added drop wise to the HBSS until the spectrophotometer yielded an absorbance of $0.045 .25 \mu \mathrm{l}$ of this bacterial preparation yields bacteria to leukocyte ratio of 1.0 to 1.5 in the final assay.

For the bactericidal assays, in order to best mimic the situation in vivo, the leukocyte concentrations of the suspensions were adjusted using a standard clinical hemocytometer to introduce the same number of leukocytes $\left(1 \times 10^{7}\right.$ leukocytes $\left./ \mathrm{ml}\right)$ for each species regardless of the initial differences in their differential leukocyte counts (Table 2).

Bactericidal activity was measured by adding HBSS (amount necessary to achieve a final incubation volume of $1.0 \mathrm{ml}$ ), normal serum $(80 \mu \mathrm{l})$, leukocytes $(400 \mu \mathrm{l})$ and then vortexed. Subsequently, human or animal serum that was obtained from a single human or animal donor (same as the leukocyte donor) was added respectively to each incubation tube. After mixing, the bacterial preparation $(25 \mu \mathrm{l})$ was added and then vortexed again. Finally, either AI-158(20 $\mu \mathrm{l}$ of a $500 \mu \mathrm{m}$ solution in $\mathrm{HBSS} / 10 \% \mathrm{DMSO}$ ) or the equivalent volume of

\begin{tabular}{|l|c|c|c|c|c|c|}
\hline & Human & Dog & Hamster & Rat & Pig & Rabbit \\
\hline $\begin{array}{l}\text { Total Leukocyte Count } \\
\left(\mathrm{x} 10^{3} / \mu \mathrm{l}\right)\end{array}$ & 5 to 11 & 6 to 16 & 5 to 10 & 6 to 18 & 7 to 20 & 7 to 15 \\
\hline PMN (including bands) & $50-70 \%$ & $55-85 \%$ & $20-30 \%$ & $10-25 \%$ & $30-55 \%$ & $20-50 \%$ \\
\hline Lymphocytes & $20-40 \%$ & $10-40 \%$ & $50-80 \%$ & $65-85 \%$ & $40-60 \%$ & $40-80 \%$ \\
\hline Monocytes & $3-9 \%$ & $2-9 \%$ & $0-3 \%$ & $0-5 \%$ & $2-10 \%$ & $1-4 \%$ \\
\hline
\end{tabular}

* Laboratory reference ranges from Colorado State University where the blood studies were conducted.

Table 2: Normal Ranges for Differential Leukocyte Counts for Different Species*

HBSS/10\% DMSO alone was added and the final mixture was then mixed via vortex on low speed. The final concentration of AI-158 in the incubation medium equaled $10 \mu \mathrm{M}$ or $7.0 \mu \mathrm{g} / \mathrm{ml}$.

To assess bactericidal activity, a time zero dilution of each tube was made by taking $50 \mu \mathrm{l}$ of sample suspension and diluting into $1.0 \mathrm{ml}$ HBSS(1:20 dilution). The assay tubes were then recapped and placed into a rotating incubator at $37^{\circ} \mathrm{C}$ for 60 minutes. While the samples were incubating, a second time zero dilution was made by taking $10 \mu \mathrm{l}$ of the 1:20 dilution into $1.0 \mathrm{ml}$ of HBSS (1:100 dilution). Then, $0.1 \mathrm{ml}$ of the 1:100 dilutions was plated onto sterile petri dishes with nutrient agar. Plates were then inverted and placed in a $37^{\circ} \mathrm{C}$ incubator for 24 hours.

After 60 minutes, assay samples are sonicated in a water bath for 10 seconds to break up the bacteria that are not killed. Subsequently, the 1:20 and 1:100 dilution was repeated and the samples were plated as before and maintained at $37^{\circ} \mathrm{C}$ for $24 \mathrm{hrs}$. After 24 hours, the bacterial colonies were counted and the plates were autoclaved and discarded. All specimens were plated and counted in triplicate. Each condition was assayed in duplicate resulting in a total of 6 measurements for each species. Since human leukocytes were used as controls for each of the species tested, data from a total of 18 measurements are reported for humans. In preliminary studies, we determined that AI-158 had no effect on bacterial growth in the absence of leukocytes (data not shown).

\section{Statistical analysis}

All statistical analyses were conducted using ANOVA with means testing using Tukey's test. Comparisons that were not statistically significant ( $\mathrm{p}$-values $\geq 0.05$ ) are identified as NS. Comparisons that were statistically significant ( $p$-values $<0.05$ or less) are individually identified in each figure.

\section{Results}

\section{Matrix degradation differs using leukocytes from different species}

In the absence of a specific stimulus, adding leukocytes from humans, dogs and rabbits produced only negligible matrix degradation compared to adding leukocytes from hamsters, rats and pigs (Figure 2, white bars). Adding LPS + FMLP increased matrix degradation over baseline levels with human, dog, hamster and rabbit leukocytes, but not with rat or pig leukocytes (Figure 2, black bars). Adding HKS increased matrix degradation over baseline levels with human, dog, or hamster leukocytes but not with rat, pig, and rabbit leukocytes (Figure 2, grey bars).

Two additional findings of interest were the following: First, the magnitude of matrix degradation after adding human leukocytes and either of the two stimuli was essentially identical (LPS + FMLP $=6.59 \pm$ $0.29 \%$ vs. HKS $=6.25 \pm 0.08 \%$ ) while matrix degradation by leukocytes from other species, specifically the dog, rat and rabbit, differed depending on the stimulus. Second, the maximum amount of matrix 


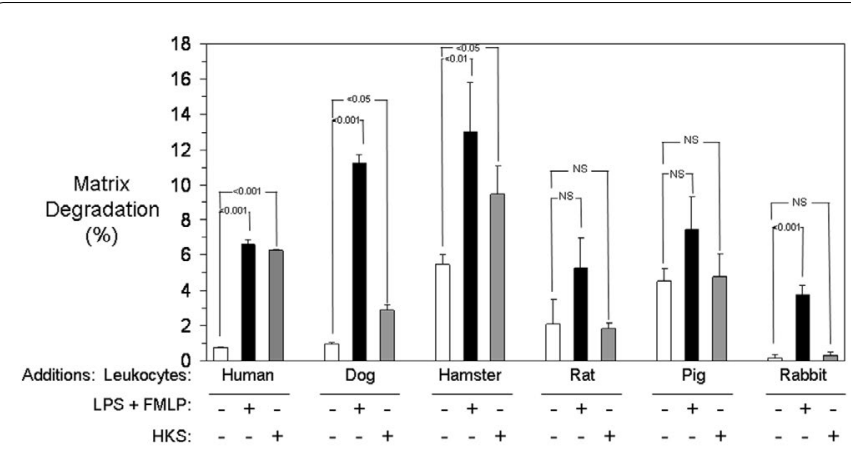

Figure 2: Leukocyte-mediated ECM degradation in response to LPS+FMLP or HKS. Leukocytes isolated from the species identified were stimulated using either a combination of LPS + FMLP (black bars) or heat-killed Staphylococcus aureus (HKS, grey bars). White bars represent the amount of matrix degradation observed after 6 hours in the absence of any added stimuli. Matrix degradation is represented as the percentage of radioactivity recovered in the supernatant relative to the total amount of radioactivity recovered after complete matrix solubalization with $2 \mathrm{M} \mathrm{NaOH}$. Each value is the mean $\pm \mathrm{SEM}$ of 6 or more measurements.

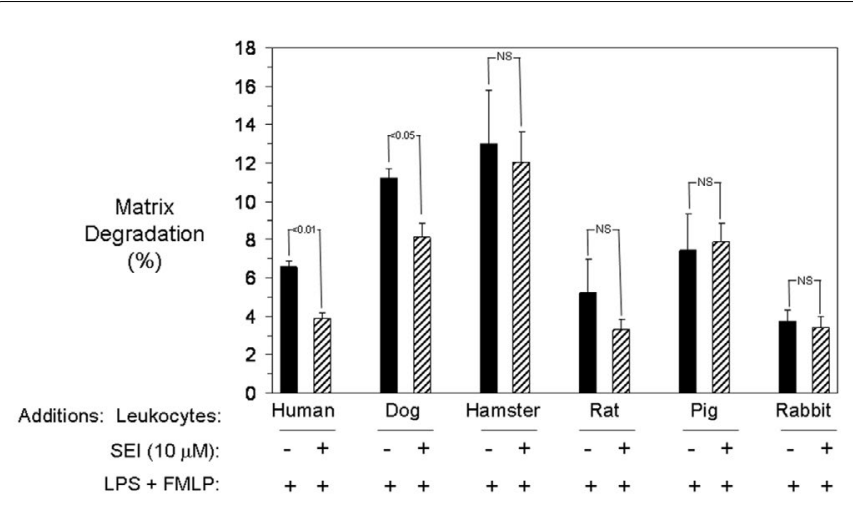

Figure 3: Effects of SEI on ECM degradation by leukocytes in response to LPS + FMLP. Leukocytes from the species identified were stimulated by LPS + FMLP either in the absence (black bars) or presence (hatched bars) of $10 \mu \mathrm{M}$ CE-2072. Matrix degradation is expressed as the percentage of radioactivity recovered in the supernatant relative to the total amount of radioactivity present in the matrix preparation. Each value is the mean \pm SEM of 6 or more measurements.

degradation by leukocytes also differed between species. Leukocytes from dogs or hamsters produced greater matrix degradation than leukocytes from humans while rat and pig leukocytes had similar maximal matrix degrading capacities compared to humans. Rabbit leukocytes were consistently at the lower end of matrix degrading capacity, regardless of stimulus. Thus, peripheral blood leukocyte mixtures from different species have different matrix degradation capacities and respond differently to microbial-derived stimuli.

\section{Matrix degradation by leukocytes from different species differs after adding a serine elastase inhibitor (SEI)}

We next assessed the effect of adding a Serine Elastase Inhibitor (SEI) on the matrix degradation caused by leukocytes from different species (Figure 3 and 4). Adding a serine elastase inhibitor decreased matrix degradation by human and dog leukocytes stimulated by LPS + FMLP---but not by leukocytes from hamsters, rats, pigs or rabbits stimulated with LPS + FMLP (Figure 3). Similarly, adding a serine elastase inhibitor decreased matrix degradation by leukocytes from humans, dogs and hamsters stimulated by HKS--- but not by leukocytes from rats, pigs or rabbits stimulated by HKS (Figure 4). In aggregate, these findings indicate that adding SEI has different inhibitory effects on matrix degradation by leukocytes from different species.

\section{Nascent bactericidal activity is equivalent for leukocytes from different species}

We next evaluated the ability of leukocytes to kill bacteria in vitro (Figure 5 and 6) focusing on the four most relevant species. In addition to human, dog and hamster leukocytes, rat leukocytes were included in the bactericidal studies because of the predominant use of rats in pathophysiology, safety, toxicity and/or interventional efficacy studies reported in the scientific literature and/or used for regulatory submissions.

Leukocytes from humans, dogs, hamsters and rats all killed E. coli (Figure 5) and S. aureus (Figure 6) in vitro. Although there appeared to be some species differences in the magnitude of bactericidal activity in vitro, none of these differences were statistically significant when comparing one species to another species.

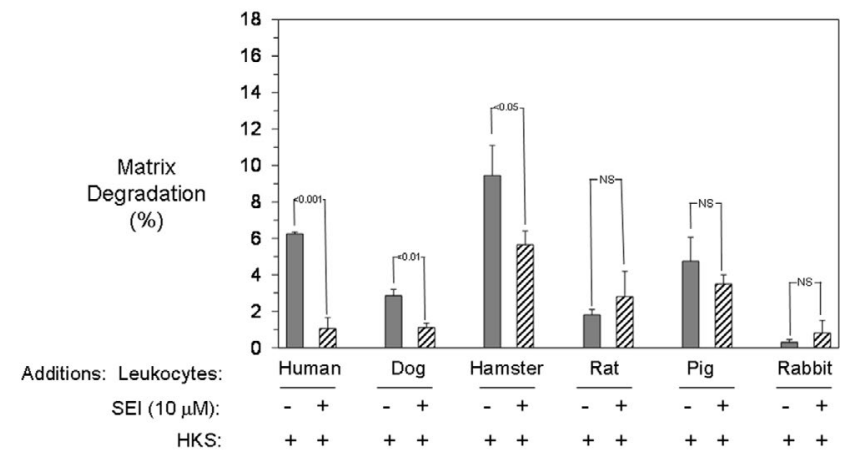

Figure 4: Effects of SEI on ECM degradation by leukocytes in response to HKS. Leukocytes from the species identified were stimulated by HKS either in the absence (grey bars) or presence (hatched bars) of $10 \mu \mathrm{M}$ CE-2072. Matrix degradation is expressed as the percentage of radioactivity recovered in the supernatant relative to the total amount of radioactivity present in the matrix preparation. Each value is the mean \pm SEM of 6 or more measurements.

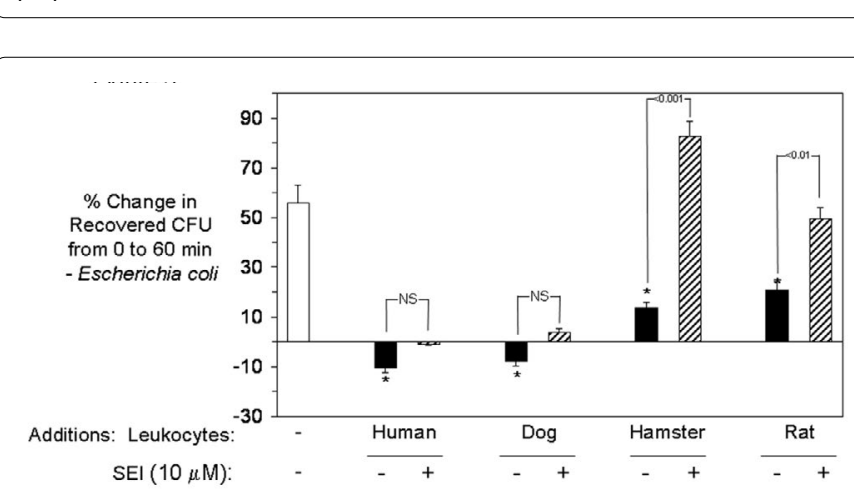

Figure 5: Effects of SEI on leukocyte-mediated bactericidal activity against Escherichia coli. Bactericidal activity is represented by the percentage change (increase or decrease) in the number of colony forming units (CFU) of $E$. coli recovered after one hour of exposure to leukocytes derived from the species identified in the absence (black bars) and presence (hatched bars) of $10 \mu \mathrm{M} \mathrm{Al}-158$ relative to the number of CFU recovered at time zero. The white bar represents the percentage change (increase) in the number of CFU recovered after one hour of incubation in the absence of any leukocytes or inhibitor relative to the number of CFU recovered at time zero. Preliminary studies demonstrated that $10 \mu \mathrm{M} \mathrm{Al}-158$ had no effect (positive or negative) on the percentage change in recovered CFU after one hour of incubation in the absence of leukocytes (data not shown). Each value is the mean \pm SEM of 6 or more measurements. ${ }^{*} p<0.001$ relative to control (white bar). 


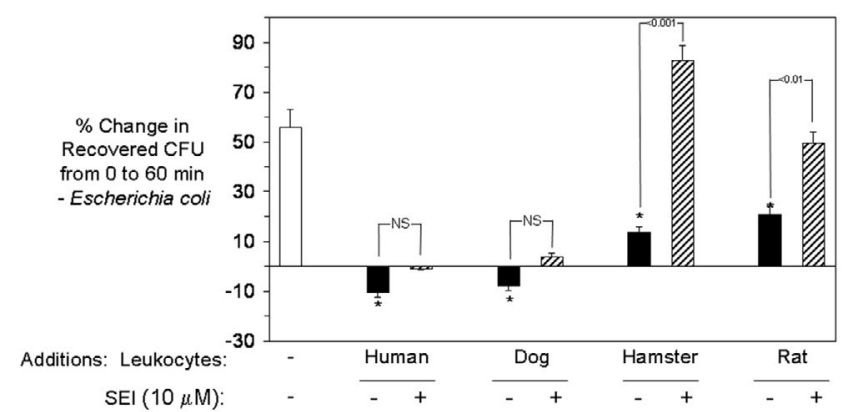

Figure 6: Effects of SEI on leukocyte-mediated Gram positive bactericidal activity against Staphylococcus aureus. Bactericidal activity is represented by the percentage change (increase or decrease) in the number of colony forming units (CFU) of $S$. aureus recovered after one hour of exposure to leukocytes derived from the species identified in the absence (black bars) and presence (hatched bars) of $10 \mu \mathrm{M} \mathrm{Al}-158$ relative to the number of CFU recovered at time zero. The white bar represents the percentage change (increase) in the number of CFU recovered after one hour of incubation in the absence of any leukocytes or inhibitor relative to the number of CFU recovered at time zero. Preliminary studies demonstrated that $10 \mu \mathrm{M} \mathrm{Al}-158$ had no effect (positive or negative) on the percentage change in recovered CFU after one hour of incubation in the absence of leukocytes (data not shown). Each value is the mean \pm SEM of 6 or more measurements. ${ }^{*} p<0.001$ relative to control (white bar).

\section{Serine elastase inhibitors have disparate effects on the bactericidal activity of leukocytes from different species}

With respect to E.coli bactericidal activity, while the presence of a serine elastase inhibitor had no effect on the killing of $E$. coli by leukocytes from humans or dogs, it completely reversed the killing of E. coli by leukocytes from hamsters and rats (Figure 5). Similarly, with respect to $S$. aureus bactericidal activity, while adding the serine elastase inhibitor had no effect on the killing of $S$. aureus by leukocytes from humans, dogs, or hamsters, adding a serine elastase inhibitor completely abrogated the ability of rat leukocytes to kill S. aureus (Figure 6).

\section{Discussion}

In the present investigation, we completed a comprehensive series of studies to begin to evaluatethe responses of different animal species with the ultimate goal being the development of models for studying leukocyte-mediated inflammatory conditions and for developing and testing serine elastase inhibitor-based interventions for acute human inflammatory conditions. Our studies involved head to head comparisons of leukocytes from human subjects and five commonly used animal species namely dogs, hamsters, rats, pigs, and rabbits. The work was prompted, in part, by a recent discovery showing that following acute inflammatory insult, the genomic responses of total blood leukocytes from mice only poorly reflect the responses of total blood leukocyte responses from human subjects [24]. This provocative observation suggests that the conventional approach of using mice to model acute inflammatory human diseases may not be ideal for some comparisons---a possibility that Seok, et al. [24] suggest might in part account for the invariably poor success rate of clinical trials in criticallyill individuals that are based on a successful therapeutic outcome in other species. However, their conclusion is based largely on differences in gene expression found in leukocytes from mice and humans rather than showing a specific species-dependent difference in function and/ or a different response to a pharmacologic intervention. Nonetheless, their concern and premise are reasonable and deserve more direct evaluation and consideration.
We found appreciable species-dependent differences in the function and response of leukocytes from different species both in the presence and absence of serine elastase inhibitors. The practical outcome is to indicate that variability exists in the matrix degradation and bactericidal activities of human leukocytes relative to those derived from various species. Furthermore, matrix degradation and antimicrobial bactericidal activities also appear to be species dependent after adding serine elastase inhibitors.

We made a number of strategic decisions in the design of our studies. First, we used complete mixtures of peripheral blood leukocytes rather than isolating a specific leukocyte type. Our rationale was based in part on the widely different total numbers and differential percentages of various leukocyte types in the peripheral blood from each species (Table 2). Furthermore, because the roles played in matrix degradation and bactericidal activity by individual leukocytes from different species is unknown, by studying the behaviors of complete mixtures of leukocytes derived from each species, we would be testing a more relevant situation approaching what occurs in vivo. Additionally, this approach eliminates concerns about the challenges of isolating and purifying specific leukocyte types from different species.

Second, we tested two distinct functions of leukocytes--. extracellular matrix degradation and gram negative and gram positive bactericidal activity---that are both relevant to human disease, Matrix degradation is a good model for assessing the critical ability of leukocytes to transmigrate from the vascular space and into tissue in response to an infection, an activity that may not only be beneficial by allowing for the killing and clearance of microbial pathogens but also detrimental by disturbing vascular integrity and causing the tissue injury, edema and organ failure that occurs in disorders such as ARDS [19-22, 32-34].

Third, we used two different stimuli of relevance to human infectious diseases to assess the ECM degrading capacities of leukocytes derived from different species. LPS + FMLP is a potent surrogate for a gram-negative type infection while HKS can be considered reasonable substitute for a gram-positive infection. This dual approach was included to emphasize that the response to different physiologically relevant stimuli may also be species dependent. Additionally, using inanimate stimuli instead of live bacteria removes the potentially confounding variable of the interaction of the pathogens with the extracellular matrix, which might complicate the observations made regarding the actions of the leukocytes themselves in response to these stimuli.

Some of the observed species differences merit mention. First, the peripheral differential blood leukocyte counts of various species differs noticeably with the differential blood leukocyte counts of dogs more closely resembling the differential blood leukocyte counts of humans than the differential blood leukocyte counts of hamsters, rats, pigs, or rabbits. More specifically, only humans and dogs have a preponderance of phagocytes (neutrophils and monocytes) compared to lymphocytes while hamsters, rats, pigs and rabbits have a predominance of lymphocytes compared to neutrophils and monocytes in their peripheral bloods. Second, adding LPS + FMLP increased the matrix degradation of leukocytes from humans, dogs, and hamsters but not rats, pigs, or rabbits. Similarly, adding HKS increased the matrix degradation by leukocytes from humans, dogs, and hamsters but not rats, pigs, or rabbits. This wide variability in the response of leukocytes to these inflammatory stimuli with respect to matrix degradation, which requires the directed release of proteolytic enzymes into the interface between the cell and vasculature, is also notable given that 
leukocytes appear to kill both gram negative and gram positive pathogens equivalently.

Third, matrix degradation and bactericidal activities of leukocytes from different species were different following addition of a highly selective Serine Elastase Inhibitors (SEI). More specifically, adding an SEI consistently decreased matrix degradation by LPS + FMLP and HKS stimulated leukocytes from humans and dogs, but not hamsters, rats, pigs and rabbits.

Fourth, adding a serine elastase inhibitor had no effect on the killing of E. coli and S. aureus by leukocytes from humans or dogs but decreased the bactericidal activity of leukocytes from hamsters (E. coli) and rats (E. coli and S. aureus). Like their effects on matrix degradation, adding a serine elastase inhibitor had different effects on the bactericidal activity of leukocytes from different species. This finding suggests that both endocytic/phagocytic pathways involved in the killing of bacteria as well as the molecular mechanisms for facilitating directed granular exocytosis required for the migration of leukocytes across endothelial barriers differ between species.

The concept that species differences might exist, especially in immune responses, is not new $[35,36]$. This possibility has been suspected for many years and is consistent with the many millions of years of evolution [37]. By way of example, species-differences have been found in A and B-defensins [38,39], corticosteroid binding globulins [40], Toll-like receptor expression [41], myocardial infarction susceptibility [42], lung inflammation following particle in sufflation [43] and many other inflammation related factors [36,37] More specifically, leukocyte function also appears impacted by species differences $[44,45]$. However, in most cases, species-difference comparisons were made inferentially rather than in head to head comparisons conducted using the same assays in the same laboratory. For example, rodents are relatively insensitive to endotoxin, so comparing the response of humans given lower doses of endotoxin to the response of rodents given higher doses of endotoxin may provide a better comparison than treating both species with the same dose of endotoxin. The parameter being measured is also important. Again, by way of example, comparing gene expression changes related to inflammatory cytokines may not produce the same conclusion as comparing actual inflammatory cytokine levels. The oxidative burst of leukocytes treated with Phorbol Myristate Acetate (PMA) is instructive. Leukocytes from rats, humans, pigs all make variable amounts of superoxide when stimulated with PMA but each leukocyte preparation was studied in different laboratories so the variation might be accounted for by actual species differences in the leukocyte responses and/or by separation and/or testing techniques. In addition, large individual to individual variations in a specific species may account for some of the reported differences [41]. Furthermore, despite the importance of using animal models for drug development and testing, little is known about the species-dependent differences in the response to pharmacologic agents like that observed in the present paper [46].

The importance of our investigation is demonstrating that differences exist in the activities and sensitivities of leukocytes from humans and other commonly used experimental species. Our results also underline the importance of making careful choices regarding which animal species should be used to model inflammatory pathophysiology before extrapolating the results to human subjects. In addition, because of the expense and time needed to develop new therapeutic agents, care must also be taken to use the most appropriate animal models when evaluating novel compounds for further development. Obviously, while it is still necessary to use animals for the early and well-controlled testing of a new pharmacologic agent before it can be advanced for testing in complex and expensive human trials, the use of an optimal animal species may ultimately guide a more successful outcome in human clinical trials and in patient care.

\section{Acknowledgements}

This work was supported in part by Contract A1066357from National Institute of Allergy and Infectious Diseases, and RO1-HL45582 from National Institutes of Health.

\section{References}

1. Blank R, Napolitano LM (2011) Epidemiology of ARDS and ALI. Crit Care Clin 27: 439-458.

2. Frutos-Vivar F, Ferguson ND, Esteban A (2006) Epidemiology of acute lung injury and acute respiratory distress syndrome. Semin Respir Crit Care Med 27: 327-336.

3. Rubenfeld GD, Caldwell E, Peabody E, Weaver J, Martin DP, et al. (2005) Incidence and outcomes of acute lung injury. N Engl J Med 353: 1685-1693.

4. The ARDS Definition Task Force (2012) The Berlin definition of ARDS. Journal of the American Medical Association307: E1-E8.

5. Donahoe M (2011) Acute respiratory distress syndrome: A clinical review. Pulm Circ 1: 192-211.

6. Ware LB (2006) Pathophysiology of acute lung injury and the acute respiratory distress syndrome. Semin Respir Crit Care Med 27: 337-349.

7. Dushianthan A, Grocott MP, Postle AD, Cusack R (2011) Acute respiratory distress syndrome and acute lung injury. Postgrad Med J 87: 612-622.

8. Bakowitz M, Bruns B, McCunn M (2012) Acute lung injury and the acute respiratory distress syndrome in the injured patient. Scand J Trauma Resusc Emerg Med 20: 54

9. Mac Sweene R, McAuleyD (2010) Pharmacological therapy for acute lung injury. Open Crit Care Med J3: 7-19.

10. Repine JE (1992) Scientific perspectives on adult respiratory distress syndrome. Lancet 339: 466-469.

11. Ganz T (2003) Defensins: antimicrobial peptides of innate immunity. Nat Rev Immunol 3: 710-720.

12. Hampton MB, Kettle AJ, Winterbourn CC (1998) Inside the neutrophil phagosome: oxidants, myeloperoxidase, and bacterial killing. Blood 92: 30073017.

13. Thomas EL, Lehrer RI, Rest RF (1988) Human neutrophil antimicrobial activity. Rev Infect Dis 10 Suppl 2: S450-456.

14. Reeves EP, Lu H, Jacobs HL, Messina CG, Bolsover S, et al. (2002) Killing activity of neutrophils is mediated through activation of proteases by $\mathrm{K}+$ flux. Nature 416: 291-297.

15. Belaaouaj A, McCarthy R, Baumann M, Gao Z, Ley TJ, et al. (1998) Mice lacking neutrophil elastase reveal impaired host defense against gram negative bacterial sepsis. Nat Med 4: 615-618.

16. Belaaouaj A, Kim KS, Shapiro SD (2000) Degradation of outer membrane protein A in Escherichia coli killing by neutrophil elastase. Science 289: 11851188.

17. Weinrauch Y, Drujan D, Shapiro SD, Weiss J, Zychlinsky A (2002) Neutrophil elastase targets virulence factors of enterobacteria. Nature 417: 91-94.

18. Cheronis JC, Rabinovitch M (2000) Serine Elastase in Inflammatory and Vascular Diseases. In: Proteases as Targets for Therapy, Handbook of Experimental Pharmacology. von der Helm K, Korant B, Cheronis JC (eds), Springer-Verlag, Heidelberg.

19. Korkmaz B, Moreau T, Gauthier F (2008) Neutrophil elastase, proteinase 3 and cathepsin G: physicochemical properties, activity and physiopathological functions. Biochimie 90: 227-242.

20. Pham CT (2006) Neutrophil serine proteases: specific regulators of inflammation. Nat Rev Immunol 6: 541-550.

21. Pham CT (2008) Neutrophil serine proteases fine-tune the inflammatory response. Int J Biochem Cell Biol 40: 1317-1333. 
Citation: Cheronis NJ, Groathouse NA, Bowen RA, Simon SR, Cheronis JC, et al. (2013) Disparate Species-Dependent Leukocyte Behavior before and after Adding an Elastase Inhibitor. J Pulm Respir Med 3: 155. doi:10.4172/2161-105X. 1000155

22. Wiedow O, Meyer-Hoffert U (2005) Neutrophil serine proteases: potential key regulators of cell signalling during inflammation. J Intern Med 257: 319-328.

23. Zeiher BG, Matsuoka S, Kawabata K, Repine JE (2002) Neutrophil elastase and acute lung injury: prospects for sivelestat and other neutrophil elastase inhibitors as therapeutics. Crit Care Med 30: S281-287.

24. Seok J, Warren HS, Cuenca AG, Mindrinos MN, Baker HV, et al. (2013) Genomic responses in mouse models poorly mimic human inflammatory diseases. Proc Natl Acad Sci U S A 110: 3507-3512.

25. Wieczorek M, Gyorkos A, Spruce LW, Ettinger A, Ross SE, et al. (1999) Biochemical characterization of alpha-ketooxadiazole inhibitors of elastases. Arch Biochem Biophys 367: 193-201.

26. Jones PA, Scott-Burden T, Gevers W (1979) Glycoprotein, elastin, and collagen secretion by rat smooth muscle cells. Proc Natl Acad Sci U S A 76: 353-357.

27. de Clerck YA, Jones PA (1980) The effect of ascorbic acid on the nature and production of collagen and elastin by rat smooth-muscle cells. Biochem $\mathrm{J} 186$ : 217-225.

28. Rinehart AR, Mallya S, Simon SR (1993) Human alpha 1-proteinase inhibitor binds to extracellular matrix in vitro. Am J Respir Cell Mol Biol 9: 666-679.

29. Werb Z, Banda MJ, Jones PA (1980) Degradation of connective tissue matrices by macrophages. I. Proteolysis of elastin, glycoproteins, and collagen by proteinases isolated from macrophages. J Exp Med 152: 1340-1357.

30. Roemer EJ,Stanton KJ, Simon SR (1994) In vitro assay systems for inflammatory cell-mediated damage to interstitial extracellular matrix. In Vitro Toxicol 7: 75-81.

31. Roemer EJ,Stanton KJ, SimonSR (1994) In vitro assay systems for cell interactions with interstitial extracellular matrix. In Vitro Toxicol7: 209-224.

32. Chua F, Laurent GJ (2006) Neutrophil elastase: mediator of extracellular matrix destruction and accumulation. Proc Am Thorac Soc 3: 424-427.

33. Carden D, Xiao F, Moak C, Willis BH, Robinson-Jackson S, et al. (1998) Neutrophil elastase promotes lung microvascular injury and proteolysis of endothelial cadherins. Am J Physiol 275: H385-392.

34. Hermant B, Bibert S, Concord E, Dublet B, Weidenhaupt M, et al. (2003) Identification of proteases involved in the proteolysis of vascular endothelium cadherin during neutrophil transmigration. J Biol Chem 278: 14002-14012.
35. Bone RC (1991) The pathogenesis of sepsis. Ann Intern Med 115: 457-469.

36. Haley PJ (2003) Species differences in the structure and function of the immune system. Toxicology 188: 49-71.

37. Mestas J, Hughes CC (2004) Of mice and not men: differences between mouse and human immunology. J Immunol 172: 2731-2738.

38. Patil AA, Cai Y, Sang Y, Blecha F, Zhang G (2005) Cross-species analysis of the mammalian beta-defensin gene family: presence of syntenic gene clusters and preferential expression in the male reproductive tract. Physiol Genomics 23: 5-17.

39. Patil A, Hughes AL, Zhang G (2004) Rapid evolution and diversification of mammalian alpha-defensins as revealed by comparative analysis of rodent and primate genes. Physiol Genomics 20: 1-11.

40. Gardill BR, Vogl MR, Lin HY, Hammond GL, Muller YA (2012) Corticosteroidbinding globulin: structure-function implications from species differences. PLoS One 7: e52759.

41. Rehli M (2002) Of mice and men: species variations of Toll-like receptor expression. Trends Immunol 23: 375-378.

42. Dewald O, Ren G, Duerr GD, Zoerlein M, Klemm C, et al. (2004) Of mice and dogs: species-specific differences in the inflammatory response following myocardial infarction. Am J Pathol 164: 665-677.

43. Carter JM, Driscoll KE (2001) The role of inflammation, oxidative stress, and proliferation in silica-induced lung disease: a species comparison. J Environ Pathol Toxicol Oncol 20 Suppl 1: 33-43.

44. Lee JJ, Jacobsen EA, Ochkur SI, McGarry MP, Condjella RM, et al. (2012 Human versus mouse eosinophils: "that which we call an eosinophil, by any other name would stain as red". J Allergy Clin Immunol 130: 572-584.

45. Isin ZhM, Suleimenov BM (1987) Comparative investigation of PMN leucocyte antimicrobial potential in animals of different species susceptibility to the plague agent. J Hyg Epidemiol Microbiol Immunol 31: 307-312.

46. Bito LZ (1984) Species differences in the responses of the eye to irritation and trauma: a hypothesis of divergence in ocular defense mechanisms, and the choice of experimental animals for eye research. Exp Eye Res 39: 807-829. 